\title{
Optimum Template Selection for Atlas-Based Segmentation
}

\section{Wu ${ }^{1}$, J. LoSurdo ${ }^{2}$, P. Lopez-Garcia ${ }^{3}$, C. S. Carter ${ }^{3}$, H. J. Aizenstein ${ }^{4}$}

${ }^{1}$ Department of Electrical \& Computer Engineering, University of Pittsburgh, Pittsburgh, PA, United States, ${ }^{2}$ Bioengineering, University of Pittsburgh, Pittsburgh, PA, United States, ${ }^{3}$ Psychiatry, University of California at Davis, Sacramento, CA, United States, ${ }^{4}$ Psychiatry, University of Pittsburgh, Pittsburgh, PA, United States

\section{Introduction}

Atlas-based segmentation has become a standard method for automatically labeling regions of interests (ROIs) in MR brain images. However normal individual variations in human brain structures present a significant challenge for atlas selection. Many methods have been explored to address this issue by constructing specific atlases to meet certain requirements. For instance, a population-based atlas is constructed from averaging individual co-registered images, which represents groupspecific features but lacks anatomical details [1]; in order to preserve the contrast and improve signal to noise (SNR), Colin27 was constructed by averaging 27 scans of the same subject co-registered to a group image [2]. In a recent study we used an atlas-based segmentation method with the Colin27 as the reference image and found overlap ratios (ORs) for the hippocampus of 57.9\% and the anterior cingulate cortex (ACC) of $47.3 \%$ [3]. We suspected that the lower OR for the ACC was due to the normal inter-subject variability in the gyral folding pattern in this structure. Because the template is a single brain image, it is unable to represent the multiple possible normal variations in brain anatomy. For instance, it has been estimated that approximately 30-60\% of the population have a paracingulate sulcus (PCS)[4], a normal variant of the ACC in which there is an additional gyral fold. The current study was designed to see if using a family of templates would improve atlas-based segmentation of the ACC.

\section{Methods}

In this study, we addressed the atlas selection problem from a different point of view. Instead of choosing a fixed atlas such as Colin27 or a population-based atlas, we used a family of templates and for each subject chose the template that gave the optimum localized registration for a specific ROI. Registration was performed using the same method as in our previous study [3], a demons-based fully deformable registration method implemented in ITK [5]. The registration accuracies of different templates were compared at a standard local region area, which was formed as the conjunction image of all of the segmented ROIs from all templates. Normalized mutual information (MI) was used as the metric to measure the local registration performance: mutual information: $I(x, y)=H(x)+H(\mathrm{y})-H(x, y)$; Normalized mutual information: $M I(x, y)=2 \cdot I(x, y) /(H(x)+H(y))$; with $H(x), H(y)$ are the entropies of subject and warped template image at the local areas. $H(x, y)$ is the joint entropy of the two local areas. In this manner, we chose the template locally optimized for each subject's ROI. Nine subjects (6 male; mean age 24.3 , range $20-32$ years; all right-handed) were scanned using a $1.5 \mathrm{~T}$ GE CVi scanner with 3DSPGR $(\mathrm{TR} / \mathrm{TE}=25 / 5 \mathrm{~ms}$; flip angle $=40^{\circ} ; \mathrm{FOV}=24 \times 18 \mathrm{~cm}$, slice thickness $=$ $1.5 \mathrm{~mm}$, and matrix size $=256 \times 192)$. Manual segmentations of the right ACC were done on all the 9 subjects and served as gold standard segmentations to evaluate the automated atlas-based segmentation results. The manual segmentations also served as atlases on the subject when used as the template. The ACC was chosen as the analyzed region because it is known to have significant individual variability; some subjects possess a paracingulate, an extra gyral fold, which makes it more difficult to automatically segment using single template.

In this experiment, the registration accuracies from the template selection based registration method were compared to the results from a single template based registration. Each of the 9 subjects was used as the template and the manually segmented ACC was used as the atlas at the registration (template $->$ the other 8 subjects). Performance of the template was evaluated using the mean overlap ratio, which measured the percentage of structure voxels that the autosegmented and the ground-truth ROIs have in common across the 8 subjects. The registration accuracy of template selection based atlas-based segmentation was measured as the mean overlap ratio across the 9 subjects.

Results

The template selection atlas-based segmentation produced significantly better mean overlap ratios for ACC segmentation across the 9 subjects than any single template segmentation. One subject, 3 template images and the MI results from the template selection model are shown in Fig. 1. It can be noted from the figure that the target subject has a paracingulate structure (in red color), and that template 2 , which has a similar paracingulate sulcus (in red) was automatically selected as the optimum template to segment the ACC on the subject using the maximum normalized mutual information between the warped image and the target subject at local areas.

Results from the optimum template and individual templates were compared. The mean ACC overlap ratio when registering using a single template ranged from $42.7 \%$ to $52.7 \%$; while the mean overlap ratio from the optimum template selection method was $54.7 \%$ (Fig. 2). As can be seen in Fig. 2, the performance of the optimum template selection method was better than the performance of any of the template candidates. A two-tailed t-test showed that the registration result from optimum template based method was significantly better than the results from single templates at $\mathrm{p}=0.0024$;

\section{Conclusion}

In this study, we found that the overlap ratio from template selection method were statistically significantly better than the results from using a single template. This template selection method is of special use to those regions such as ACC, which have natural variation across different subjects. A single template could not address these differences. This higher registration accuracy with better template selection is achieved at the cost of higher computation load, by requiring multiple template registrations. This method chooses the optimum template for a specific subject and a specific ROI using normalized mutual information from local areas; and it can be accommodated into alternate available registration pathways such as SPM or AIR.

References

[1] Thompson PM et al. Human Brain Mapp. 2000; 9:81-92. [2] Holmes CJ et al. J Comput Assist Tomogr. 1998; 22:324-333. [3] Wu M. et al. p.1566 ISMRM 2005. [4] Paus, T. et al. Cereb Cortex. 1996; 6(2), 207-14. [5] Yoo T. ISBN: 1-56881-217-5. Wellesey, MA: AK Peters Ltd. 